\title{
Association Between Modified Nordic Diet With Sleep Quality and Circadian Rhythm in Overweight and Obese Woman: A Cross Sectional Study
}

ahmad mousavi

Islamic Azad University Science and Research Branch https://orcid.org/0000-0001-8154-5325

Atieh Mirzababaei

Tehran University of Medical Sciences

Farideh Shiraseb

Tehran University of Medical Sciences

Khadijeh Mirzaei ( $\nabla$ mina_mirzaei101@yahoo.com )

Tehran University of Medical Sciences

\section{Research Article}

Keywords: modified Nordic diet, diet quality, sleep quality, circadian rhythm

Posted Date: June 4th, 2021

DOI: https://doi.org/10.21203/rs.3.rs-317678/v1

License: (a) (i) This work is licensed under a Creative Commons Attribution 4.0 International License. Read Full License 


\section{Abstract}

Objective

Previous studies have shown the association between diet quality and sleep quality. the objective of this study was to observe the association between modified Nordic diet with sleep quality and circadian rhythm in overweight and obese woman.

Methods

We enrolled 399 overweight and obese women (body mass index (BMI): $25-40 \mathrm{~kg} / \mathrm{m}^{2}$ ) aged 18-48 years in cross-sectional study. For each participant anthropometric measurements, biochemical test and food intake were evaluated. Sleep quality and circadian rhythm was measured by Pittsburgh Sleep Quality Index (PSQI) and Morning Evening Questionnaire (MEQ) questionnaire. Modified Nordic diet score was measured using a validated 147-item food frequency questionnaire (FFQ).

Results

Among all participants, Overall, $51.7 \%$ of the subjects were good sleepers (the Pittsburgh Sleep Quality Index (PSQI) < 5) while 48.3\% were poor sleepers (PSQI $\geq 5$ ). Moreover, participants were divided into 5 groups of MEQ namely, completely morning $8(2.4 \%)$, rarely morning $82(24.8 \%)$, normal $196(59.2 \%)$, rarely evening $43(13 \%)$, completely evening $2(0.6 \%)$. After controlling for confounder there was a significant association between the poor sleep quality and modified Nordic diet $(\mathrm{OR}=0.80, \% 95 \mathrm{Cl}=0.66-0.98$, $\mathrm{P}=0.01)$. Moreover, a significant positive association was observed between the completely morning and modified Nordic diet $(\mathrm{OR}=1.80, \% 95 \mathrm{Cl}=0.54-6.00, \mathrm{P}=0.03)$ and also a significant reverse association was observed between completely evening type and modified Nordic diet $(\mathrm{OR}=0.16, \% 95 \mathrm{Cl}=0.002-5.41$, $\mathrm{P}=0.02)$.

Conclusions

The present study indicate that higher adherence to a modified Nordic diet decreases poor sleep quality. Also, the completely morning type associated with higher adherence to a modified Nordic diet and completely evening type associated with lower adherence to a modified Nordic diet.

Levels of Evidence

Level IV, evidence obtained from with multiple time series analysis.

\section{Introduction}

The prevalence of obesity has progressively risen in all parts of the world during the last decades and has become a main public health concern, in the last two decades, a significantly increasing trend in obesity was observed (1). According to the National Health and Nutrition Examination and Survey in 2017-2018, 
the age-adjusted prevalence of obesity in adults was $42.4 \%$, and there were no significant differences between men and women among all adults or by age group (1).

The global epidemic of obesity results from a combination of genetic susceptibility, increased availability of high-energy foods and decreased requirement for physical activity in modern society (2). Obesity is reported to be related to impaired mental health such as anxiety, stress, depression and low quality of sleep $(3,4)$. Over the past 60 years, sleep duration was reported to reduce from $9 \mathrm{~h}$ per day to $7 \mathrm{~h}$ per day (5) and also insomnia is a common clinical condition, characterized by difficulty initiating or maintaining sleep, with a prevalence of $10 \%-20 \%$ (6). Some studies have reported that lower sleep quality can be related with the higher rate of obesity and this phenomenon has become more common over the past decade.in addition, it was reported that sleep deficiency and lower diet-quality are related with chronic diseases including diabetes mellitus and metabolic syndrome (7-9). The Nordic diet is a dietary pattern referring to consumption of traditional foods from the Nordic countries (the Scandinavian region), including whole grains, fruits (such as apples, pears, and berries), low-fat dairy products, fatty fish such as salmon, cabbage and root vegetables (10). Studies have reported that the Nordic diet can decreases the weight (11-14). Almost all living organisms have evolved autoregulatory transcriptional-translational feedback loops that produce oscillations with a period of approximately24-h. These endogenous time keeping mechanisms are called circadian clocks. The main function of these circadian clocks is to drive overt circadian rhythms in the physiology of the organisms to ensure that main physiological functions are in synchrony with the external environment. Disruption of circadian rhythms caused by genetic or environmental factors has long-term consequences for metabolic health. Of relevance, host circadian rhythmicity and lipid metabolism are increasingly recognized to cross-regulate and the circadian clocklipid metabolism interplay may involve in the development of obesity (15). Sleep problems are considered an emerging global epidemic $(16,17)$. Studies have reported an association between sleep problems and obesity $(18,19)$ and sleep problems contribute to obesity, as changes that lead to weight gain shorter duration and poorer quality of sleep lead to behavioral, metabolic, and endocrine $(20,21)$ and also disturbing circadian rhythms were associated with elevated risk for obesity $(15,22)$. Some studies have reported diets were positively associated with sleep quality $(23,24)$ however a study has reported the Nordic diet has no association with sleep quality (25). Thus, this study was performed to assed the association between modified Nordic diet with sleep quality and circadian rhythm in overweight and obese woman.

\section{Method}

\section{Study population}

This cross-sectional study was conducted in 399 women who were referred to health centers in Tehran, Iran in 2017 to 2019 years. All subjects were randomly recruited from individuals referring to health centers in Tehran during. Inclusion criteria were age 18-48 years, being overweight or obese (body mass index (BMI): $25-40 \mathrm{~kg} / \mathrm{m} 2$ ) without any change in weight in recent year, absence of any acute or chronic infection, no alcohol or drug or supplement abuse, no history of hypertension, and not being pregnant. 
Based on exclusion criteria, subjects that consumed less than $800 \mathrm{kcal}$ and more than $4200 \mathrm{kcal}$ were excluded from the study. Written informed consent was obtained from all participants before taking part in the study. The study protocol was approved by the local ethical committee of Tehran University of Medical Sciences (Ethics number: IR.TUMS.MEDICINE.REC.1399.637).

\section{Evaluation of food intake}

To assess the dietary intake of participants, a semi-quantitative food frequency questionnaire) FFQ) with 147 Iranian food items, containing a list of foods with standard serving sizes was used. FFQ assesses the usual food intake over the previous year. The high reliability and validity of the FFQ have been confirmed previously (26). All FFQ and demographic questionnaires were completed by trained nutritionists. The energy of food consumed was evaluated using Nutritionist 4 software. The Nordic diet is a dietary pattern referring to consumption of traditional foods from the Nordic countries (the Scandinavian region), including whole grains, fruits (such as apples, pears, and berries), low-fat dairy products, fatty fish such as salmon, cabbage and root vegetables. The modified Nordic diet score was based on: (1) rye and wholegrain breads with a median of 90, (2) oatmeal (chickpea, lentil, bean, oat, frumenty, soybean, split pea, vicia faba and mung bean) with a median of 20, (3) cabbages and vegetables (cucumber, lettuce, celery, tomato, zucchini, raw and boiled spinach, bell pepper and leafy vegetables) with a median of 132, (4) apples, pears and high antioxidant fruits (apple, apple juice, peach, strawberry, nectarine, pear, persimmon, apricot, dry apricot, mulberries, dry mulberries, plum and dry plum) with a median of $97,(5)$ root vegetables (potato, raw and boiled carrot, garlic, onion, and turnip) with a median of 41 and (6) fish (fish conserved in salt and oil and other fish) with a median of 2. We calculated the median consumption of these food groups according to the FFQ. Consumption above and below median intake were given 1 and 0 points, respectively. The score of each group was summed and were classified: 0-1 point for low adherence, 2-3 points for medium adherence and 4-6 points for high adherence (10).

\section{Assessment of sleep quality}

The Pittsburgh Sleep Quality Index (PSQI) was applied to subjectively measure the sleep quality of participants. It evaluates usual sleep habits during the past month. Total scores could range from 0 to 21 , with a global sum of " 5 ” or greater indicates poor sleep quality (27).

\section{Assessment of circadian rhythm}

The morning evening questionnaire (MEQ) was applied to asses circadian rhythm. The sum gives a score ranging from 16 to 86; scores of 41 and below indicate "evening types", scores of 59 and above indicate "morning types", scores between 42-58 indicate "intermediate types (28).

\section{Assessment of other variables}

Demographic questionnaire including job status, educational level, marital status, economic status was completed by trained nutritionists.

\section{Assessment of physical activity}


Physical activity levels were assessed using a 7-item International Physical Activity Questionnaire (IPAQ) and the results recorded as metabolic equivalent hours per week (MET.h week - 1) (29).

\section{Assessment of anthropometric measurement}

The weight of the individuals was measured with the use of a digital scale (Seca, Hamburg, Germany) in light clothing and without shoes with precision near to $0.1 \mathrm{~kg}$. The height of participants was evaluated by a seca stadiometer, with exactness close to $0.1 \mathrm{~cm}$. BMI was calculated as weight (kg)/hieght2 (m). Waist (WC) and hip circumference ( $\mathrm{HC})$ were measured in the smallest girth and the largest girth, respectively, with accuracy nearest to $0.1 \mathrm{~cm}$.

\section{Statistical analysis}

A normal distribution of data was established by the Kolmogorov-Smirnov test. We divided the modified Nordic diet score into three tertiles and general characteristics across tertiles of the modified Nordic diet score were indicated as mean \pm standard deviations (SD) for continuous variables and categorical variables were expressed as numbers and percentages. One-way analysis of variance and chi-square test was used for quantitative and qualitative variables respectively. Post-Hoc Multiple Comparison analysis by Bonferroni was used to demonstrate the significant differences mean between groups: analysis of covariance (ANCOVA) was used to identify dietary intake differences between tertiles of the modified Nordic diet score and all values were adjusted for energy intake, BMI, age, physical activity. Moreover, we used Multinomial logistic regressions to calculate odds ratio (OR) and 95\% Cl for circadian rhythm and sleep quality across tertile of modified Nordic diet score in crude and multivariable-adjusted models (adjusted for BMI, age, physical activity, energy intake, economic status, marital status, occupation, education, housing status, supplement consumption). All statistical analyses were done by using the Statistical Package for Social Sciences (version 22; SPSS Inc.). P 0.05 was considered to be statistically significant and $\mathrm{P}=0.06$ was considered to be statistically marginal significant.

\section{Results}

\section{Study population characteristic}

A total of 399 women aged 18-48 years, participated in this study. The quantitative and qualitative characteristics of the study subjects were shown across the tertiles modified Nordic diet shown in Table 1. At this study population the mean and standard deviation (SD) age and BMI of participants were $36.61 \pm 9.11$ year and $31.25 \pm 4.30 \mathrm{~kg} / \mathrm{m}^{2}$ respectively. Moreover, participants were divided into 5 groups of MEQ namely, completely morning $8(2.4 \%)$, rarely morning $82(24.8 \%)$, normal $196(59.2 \%)$, rarely evening $43(13 \%)$, completely evening $2(0.6 \%)$, respectively. Sleep quality was classified into two groups: good sleep quality 166(51.7\%), and poor sleep 155(48.3\%). The economic status of the study population in upper tertile was better than lower tertile, also in top tertile, more people get married than lower.

\section{Study population characteristic among tertiles of modified Nordic diet score}


After controlling potential confounders (age, physical activity (PA), total energy intake, and BMI) with ANCOVA analysis a significant mean difference was observed among tertiles of the modified Nordic score in terms of physical activity $(P=0.03)$ this means difference was between $T 1$ and $T 2$ according to Bonferroni post hoc analysis so that physical activity mean was more in $T 2$ than $T 1$ and terms of HDL ( $P$ $=0.04$ ) this mean difference was between T1 and T3 according to Bonferroni post hoc analysis so that HDL mean was more in $T 3$ than T1. There were marginally significantly for economic status variable $(P=$ 0.05).

\section{Dietary intake of study subject across tertiles of modified Nordic diet score}

modified Nordic diet components, macronutrient, and micronutrient across tertiles of modified Nordic diet score were presented in Table 2. After adjustment with potential confounders (total energy intake, physical activity, BMl, and age) there have shown the participant with high modified Nordic diet adherence (T3) than low modified Nordic diet adherence (T1) consumed a significantly higher amount of protein, Eicosapentaenoic acid (EPA), Docosahexaenoic acid (DHA), Potassium, Magnesium, Zinc, Iron, vitamin $B 6$, vitamin $C$, Caffeine $(P<0.001)$, fat $(P=0.002)$, calcium $(P=0.003)$ but carbohydrate was marginally significant $(P=0.05)$ in modified Nordic diet components. As we expected, across tertiles, significantly higher consumption of fish, root vegetable, cabbages, whole grain, oatmeal, rye bread, apple pear, vegetables $(P<0.001)$, red meat $(P=0.01)$, legumes $(P<0.001)$, fruits and Sugar-sweetened beverages $(P=0.09)$ were presented in highest levels of adherence to the modified Nordic diet.

\section{Circadian rhythm and sleep quality across tertiles of modified Nordic diet score}

The relationship between circadian rhythm and sleep quality with modified Nordic diet in crude and adjusted models was shown in Table 3. Model 1 was adjusted for energy intake, BMI, age, physical activity. Model 2 was further adjusted for economic status, job status, marital status, education. Model 3 was adjusted for homeownership, supplement consumption, caffeine consumption. After adjusting for homeownership, supplement consumption, caffeine, a marginally significant difference was observed between the T1 and T3 according to Bonferroni post hoc in terms of sleep quality in model $3(P=0.05)$ so that sleep quality mean was more in T1 than T3. Moreover, the significant difference was not observed between tertiles in model 1 and model 2 in sleep quality and any models in circadian rhythm.

\section{The association between sleep quality and circadian rhythm with modified Nordic diet score}

The association between sleep quality and circadian rhythm with modified Nordic diet was shown in the crude and adjusted regression models in Table 4. A significant reverse association was observed between the poor sleep quality and modified Nordic diet in the crude model $(\mathrm{OR}=0.81, \% 95 \mathrm{Cl}=0.68-0.98, \mathrm{P}=$ 0.02 ) that remained significant after controlling confounders (economic status energy intake, $\mathrm{BMI}$, age, physical activity) in the adjusted model $(\mathrm{OR}=0.80, \% 95 \mathrm{Cl}=0.66-0.98, \mathrm{P}=0.01)$. A significant positive 
association was observed between the completely morning type and modified Nordic diet in the adjusted model $(\mathrm{OR}=1.80, \% 95 \mathrm{Cl}=0.54-6.00, \mathrm{P}=0.03)$, and also a significant reverse association was observed between completely evening type and modified Nordic diet in the adjusted model $(\mathrm{OR}=0.16, \% 95 \mathrm{Cl}=$ $0.002-5.41, \mathrm{P}=0.02)$.

\section{Discussion}

The objective of this study was to observe the association between modified Nordic diet with sleep quality and circadian rhythm in overweight and obese woman. Our hypothesis was that a higher adherence to the modified Nordic diet score, which represents a healthy dietary pattern, is connected with better sleep quality over time. This result adds a new insight into the relationship and possible pathways between sleep and diet.

In present study, the results showed a reverse association between adherence to modified Nordic diet and poor sleep quality status. Moreover, a significant positive association was observed between the completely morning and modified Nordic diet. also, a significant reverse association was observed between completely evening type and modified Nordic diet.

Previous finding on the correlation of diets with sleep quality and circadian rhythm has been obtained mostly from cross sectional studies. One study shows short sleep duration combined with poor sleep quality is associated with low adherence to a Mediterranean diet and Nordic diet (25).

Melatonin could modulate circadian rhythm and improve sleep disorders (30-32). The interpretation is that melatonin weakens the circadian signal from the suprachiasmatic nuclei (SCN), promoting heat loss which induces sleepiness via the preoptic area of the anterior hypothalamus (33). It has been proved that the melatonin concentration in human serum could significantly increase after consumption of melatonin containing food like fruits and vegetable (34). In animal foods, melatonin concentrations were found higher in fish than those in meat (35). Other research showed calcium along with magnesium and potassium act neuromodulators in the sleep/wake cycle and may play an important role in melatonin production through the activation of tryptophan hydroxylase $(36,37)$. Many micronutrients play a role in sleep and circadian regulation, including those that support melatonin synthesis (folate, vitamin B6, zinc) (38). a study shows a greater percentage of all adults reporting short sleep had inadequate intake of copper, folate, iron, magnesium, riboflavin, zinc, and vitamins A, C and K (39). Intracellular magnesium concentration acts as a cell-autonomous timekeeping component to determine key clock properties in human cell lines and unicellular alga (40). In addition, magnesium has an essential role in ion transport and electrical conductivity, which facilitate N-methyl-D-aspartic acid (NMDA) receptor function, an important sleep regulator (41). Magnesium may also play a role in melatonin synthesis as a cofactor for serotonin $\mathrm{N}$ acetyltransferase (arylalkylamone- $\mathrm{N}$-acetyltransferase; AANAT), which facilitates the conversion of serotonin to $\mathrm{N}$-acetylserotonin, the rate limiting step in melatonin synthesis (42). In one study shows less calcium intake associated with greater difficulty falling asleep and less carbohydrate intake associated significantly with greater difficulty maintaining asleep and less potassium intake 
associated with greater daytime sleepiness and less calcium and vitamin C intake associated significantly with greater non-restorative sleep (43). Fewer sleep difficulties associated with increased calcium may have been a result of the effects of calcium on lowering blood pressure (44). Animal models of melatonin-proficient $(\mathrm{C} 3 \mathrm{H})$ and melatonin-deficient $(\mathrm{C} 57 \mathrm{BL})$ mice are often used to investigate the role of melatonin in circadian rhythms. In one study, a study was performed on three clock gene proteins, PER1, BMAL1 and CRY2, in the adrenal cortex of mice and medulla, and the results showed that $\mathrm{C} 3 \mathrm{H}$, PER1 and CRY2 mice reached a maximum in the light phase while BMAL1 peaked in the dark phase, these three clock gene protein levels showed day / night changes in both the adrenal and medullary cortex. Similar patterns were shown in the adrenal cortex of C57BL mice, but in the adrenal cortex of C57BL mice, the clock gene protein level was consistently lower than that of $\mathrm{C} 3 \mathrm{H}$ mice and did not change over time (45). The effect of polyphenols on sleep measures may be through their antioxidant content reducing oxidative stress and improving sleep quality (46). St-onge suggested that plant based diets may improve sleep quality (47). In one study, polyphenol-rich food supplement was able to significantly improved sleep quality by $43 \%(48)$. A study shows inverse association between fruits and vegetable consumption and poor sleep quality (49). A study shows low intake of vegetables and fish, high intake of confectionary and noodles and unhealthy eating habits were independently associated with poor sleep quality (50). The first potential mechanism of how polyphenols from fruits and vegetables consumption may affect sleep measures is through the gut-brain axis via serotonin and $\gamma^{-}$ aminobutyric acid (GABA) receptors, consequently affecting nocturnal secretion of melatonin (51). Polyphenols in red cabbage extracts decreased sleep onset latency (SOL) and increased sleep duration via y aminobutyric acid GABA receptors in mice (52). Low protein intake ( $<16 \%$ of energy from protein) was associated with poor quality of sleep and marginally associated with difficulty initiating sleep, whereas high protein intake (>19\% of energy from protein) was associated with difficulty maintaining sleep. low carbohydrate intake ( $<50 \%$ of energy from carbohydrate) was marginally associated with difficulty maintain sleep and when stratified by sex, these association were significant in men not in women (53). Higher consumption of meat was also associated with incidence of snoring and poor general sleep quality (50). An increase in fish intake in associated with further improvement the quality of sleep (54). Dietary fat and protein and too much smaller carbohydrate stimulate the release of cholecystokinin (CCK) in duodenum and jejunum (55). Post prandial release of CCK induce sleepiness in healthy adult 2 to 3 hours after high fat and low carbohydrate meal (56). Finally, we can say that in this study, the reason for the effect of modified Nordic diet to increase the quality of sleep due to more significant consumption of fish, root vegetable, cabbages, apple pear, vegetables, fruits, Eicosapentaenoic acid (EPA), Docosahexaenoic acid (DHA), potassium, magnesium, calcium, zinc, iron, B6, vitamin C, fat, carbohydrate.

\section{What is already known on this subject?}

Recent studies show that modified Nordic diet has emerged as a healthy eating option. Before present study, very limited studies conducted the association between modified Nordic diet and sleep. The reason we conduct the association between the modified Nordic diet and sleep is the reduction of sleep duration over the past 60 years (5) and also insomnia is a common clinical condition, characterized by difficulty 
initiating or maintaining sleep, with a prevalence of 10\%-20\% (6). Data from current study suggest those who had good quality sleep had higher adherence to a modified Nordic diet.

\section{What does this study add?}

No previous studies have been conducted relating modified Nordic diet and sleep in the Iranian population. We provide evidence that it may be one of the better predictors of the relationship between diet and sleep.

\section{Limitations and strengths}

It was cross-sectional; hence causality cannot be evaluated. Small population and the same-sex sample also limit the statistical power. Dietary assessment by FFQ questionnaire can result over- or underreporting food intake. Due to low adherence to Nordic diet in Iranian population, we could not use the original Nordic diet scored and we replaced the modified Nordic diet score. Due to the different culture and dietary intakes, our results are not possible to generalize through the country. No previous studies have been conducted association between modified Nordic diet with sleep quality and circadian rhythm in the Iranian population. We provide evidence that it may be one of the better predictors of association between diet and sleep quality.

\section{Conclusion}

We found that lower odds of poor sleep quality were associated with higher adherence to a modified Nordic diet. Also, in circadian rhythm we found that the completely morning type associated with higher adherence to a modified Nordic diet and completely evening type associated with lower adherence to a modified Nordic diet. Our results suggest that the modified Nordic diet plays a role in poor sleep quality and circadian rhythm in overweight and obese women. Clinical trials should be conducted to clarify the impact of higher adherence of modified Nordic diet on better quality of sleep. Since this is the first study that considered modified Nordic diet as a diet-quality tool measurement, further studies are required to establish these findings through replication in more diverse populations and at last to confirm this correlation.

\section{Abbreviations}

PSQI: Pittsburgh sleep quality Index, MEQ: Morning evening questionnaire

FFQ: Food frequency questionnaire, IPAQ: International Physical Activity Questionnaire

WC: Waist circumference, HC: hip circumference, EPA: Eicosapentaenoic acid, DHA: Docosahexaenoic acid, SCN: suprachiasmatic nuclei, NMDA: N-methyl-D-aspartic acid

GABA: $y$-aminobutyric acid, SOL: sleep onset latency, CCK: cholecystokinin 


\section{Declarations}

Acknowledgements: The authors are thankful to all participants and large team working on this project in different provinces. We appreciate Tehran University of Medical Sciences (Project number: 98-3-21246721) for supporting this project. All authors have read the journal's authorship agreement and the manuscript has been reviewed and approved by all named authors. We thank the editor and anonymous reviewers whose comments have greatly improved this manuscript.

Funding: This study was supported financially by Tehran University of Medical Sciences: Grant ID: 98-3212-46721

Conflict of interest: All authors declare that they have no potential conflicts of interest. Ethical approval the study protocol was approved by the Ethics Commission of Tehran University of medical sciences (Ethics number: IR.TUMS.MEDICINE.REC.1399.637).

Informed consent: A written informed consent was signed by all individuals.

\section{References}

1. Hales CM, Carroll MD, Fryar CD, Ogden CL. Prevalence of obesity and severe obesity among adults: United States, 2017-2018. 2020.

2. Bray GA. Medical consequences of obesity. The Journal of Clinical Endocrinology \& Metabolism. 2004;89(6):2583-9.

3. Marshall NS, Glozier N, Grunstein RR. Is sleep duration related to obesity? A critical review of the epidemiological evidence. Sleep medicine reviews. 2008;12(4):289-98.

4. Dimov S, Mundy LK, Bayer JK, Jacka FN, Canterford L, Patton GC. Diet quality and mental health problems in late childhood. Nutritional neuroscience. 2019:1-9.

5. Crispim CA, Zalcman I, Dáttilo M, Padilha HG, Edwards B, Waterhouse J, et al. The influence of sleep and sleep loss upon food intake and metabolism. Nutrition Research Reviews. 2007;20(2):195-212.

6. Merrigan JM, Buysse DJ, Bird JC, Livingston EH. Insomnia. Jama. 2013;309(7):733-.

7. Knutson KL, Van Cauter E. Associations between sleep loss and increased risk of obesity and diabetes. Annals of the New York Academy of Sciences. 2008;1129:287.

8. Choi J-K, Kim M-Y, Kim J-K, Park J-K, Oh S-S, Koh S-B, et al. Association between short sleep duration and high incidence of metabolic syndrome in midlife women. The Tohoku journal of experimental medicine. 2011;225(3):187-93.

9. Ayas NT, White DP, Manson JE, Stampfer MJ, Speizer FE, Malhotra A, et al. A prospective study of sleep duration and coronary heart disease in women. Archives of internal medicine. 2003;163(2):2059.

10. Daneshzad E, Emami S, Mofrad MD, Saraf-Bank S, Surkan PJ, Azadbakht L. Association of modified Nordic diet with cardiovascular risk factors among type 2 diabetes patients: a cross-sectional study. 
Journal of cardiovascular and thoracic research. 2018;10(3):153.

11. Darwiche G, Höglund P, Roth B, Larsson E, Sjöberg T, Wohlfart B, et al. An Okinawan-based Nordic diet improves anthropometry, metabolic control, and health-related quality of life in Scandinavian patients with type 2 diabetes: a pilot trial. Food \& nutrition research. 2016;60(1):32594.

12. Poulsen SK, Crone C, Astrup A, Larsen TM. Long-term adherence to the New Nordic Diet and the effects on body weight, anthropometry and blood pressure: a 12-month follow-up study. European journal of nutrition. 2015;54(1):67-76.

13. Fritzen AM, Lundsgaard A-M, Jordy AB, Poulsen SK, Stender S, Pilegaard H, et al. New Nordic DietInduced Weight Loss Is Accompanied by Changes in Metabolism and AMPK Signaling in Adipose Tissue. The Journal of Clinical Endocrinology \& Metabolism. 2015;100(9):3509-19.

14. Poulsen SK, Due A, Jordy AB, Kiens B, Stark KD, Stender S, et al. Health effect of the New Nordic Diet in adults with increased waist circumference: a 6-mo randomized controlled trial. The American journal of clinical nutrition. 2014;99(1):35-45.

15. Li Y, Ma J, Yao K, Su W, Tan B, Wu X, et al. Circadian rhythms and obesity: Timekeeping governs lipid metabolism. Journal of Pineal Research. 2020;69(3):e12682.

16. Peltzer K, Pengpid S. Nocturnal sleep problems among university students from 26 countries. Sleep and Breathing. 2015;19(2):499-508.

17. Stranges S, Tigbe W, Gómez-Olivé FX, Thorogood M, Kandala N-B. Sleep problems: an emerging global epidemic? Findings from the INDEPTH WHO-SAGE study among more than 40,000 older adults from 8 countries across Africa and Asia. Sleep. 2012;35(8):1173-81.

18. Piovezan RD, Hirotsu C, Moizinho R, de Sá Souza H, D'Almeida V, Tufik S, et al. Associations between sleep conditions and body composition states: results of the EPISONO study. Journal of cachexia, sarcopenia and muscle. 2019;10(5):962-73.

19. Sa J, Choe S, Cho B-y, Chaput J-P, Kim G, Park C-H, et al. Relationship between sleep and obesity among US and South Korean college students. BMC Public Health. 2020;20(1):96.

20. Spaeth AM, Dinges DF, Goel N. Effects of experimental sleep restriction on weight gain, caloric intake, and meal timing in healthy adults. Sleep. 2013;36(7):981-90.

21. Beccuti G, Pannain S. Sleep and obesity. Current opinion in clinical nutrition and metabolic care. 2011;14(4):402.

22. Engin A. Circadian rhythms in diet-induced obesity. Obesity and lipotoxicity: Springer; 2017. p. 19-52.

23. Muscogiuri G, Barrea L, Aprano S, Framondi L, Di Matteo R, Laudisio D, et al. Sleep quality in obesity: does adherence to the mediterranean diet matter? Nutrients. 2020;12(5):1364.

24. Bazyar H, Javid AZ, Dasi E, Sadeghian M. Major dietary patterns in relation to obesity and quality of sleep among female university students. Clinical Nutrition ESPEN. 2020;39:157-64.

25. Theorell-Haglöw J, Lemming EW, Michaëlsson K, Elmståhl S, Lind L, Lindberg E. Sleep duration is associated with healthy diet scores and meal patterns: results from the population-based EpiHealth study. Journal of Clinical Sleep Medicine. 2020;16(1):9-18. 
26. Esmaillzadeh A, Mirmiran P, Azizi F. Whole-grain intake and the prevalence of hypertriglyceridemic waist phenotype in Tehranian adults. The American journal of clinical nutrition. 2005;81(1):55-63.

27. Dj B, Reynolds C, Monk T, Berman S, Kupfer D. The Pittsburgh Sleep Quality Index: a new instrument for psychiatric practice and research. Psychiatry Res. 1989;28(2):193-213.

28. Horne JA, Östberg 0 . A self-assessment questionnaire to determine morningness-eveningness in human circadian rhythms. International journal of chronobiology. 1976.

29. Ainsworth BE, Haskell WL, Whitt MC, Irwin ML, Swartz AM, Strath SJ, et al. Compendium of physical activities: an update of activity codes and MET intensities. Medicine and science in sports and exercise. 2000;32(9; SUPP/1):S498-S504.

30. Wehr TA, Duncan WC, Sher L, Aeschbach D, Schwartz PJ, Turner EH, et al. A circadian signal of change of season in patients with seasonal affective disorder. Archives of general psychiatry. 2001;58(12):1108-14.

31. Lundmark PO, Pandi-Perumal S, Srinivasan V, Cardinali D, Rosenstein R. Melatonin in the eye: implications for glaucoma. Experimental eye research. 2007;84(6):1021-30.

32. Hardeland R. Neurobiology, pathophysiology, and treatment of melatonin deficiency and dysfunction. The Scientific World Journal. 2012;2012.

33. Cajochen C, Kräuchi K, Wirz-Justice A. Role of melatonin in the regulation of human circadian rhythms and sleep. Journal of neuroendocrinology. 2003;15(4):432-7.

34. Meng X, Li Y, Li S, Zhou Y, Gan R-Y, Xu D-P, et al. Dietary sources and bioactivities of melatonin. Nutrients. 2017;9(4):367.

35. Tan DX, Zanghi BM, Manchester LC, Reiter RJ. Melatonin identified in meats and other food stuffs: potentially nutritional impact. Journal of pineal research. 2014;57(2):213-8.

36. Barbosa R, Scialfa JH, Terra IM, Cipolla-Neto J, Simonneaux V, Afeche SC. Tryptophan hydroxylase is modulated by L-type calcium channels in the rat pineal gland. Life sciences. 2008;82(9-10):529-35.

37. Kuhn DM, O'Callaghan JP, Juskevich J, Lovenberg W. Activation of brain tryptophan hydroxylase by ATP-Mg2+: dependence on calmodulin. Proceedings of the National Academy of Sciences. 1980;77(8):4688-91.

38. Peuhkuri K, Sihvola N, Korpela R. Dietary factors and fluctuating levels of melatonin. Food \& nutrition research. 2012;56(1):17252.

39. Ikonte CJ, Mun JG, Reider CA, Grant RW, Mitmesser SH. Micronutrient inadequacy in short sleep: Analysis of the NHANES 2005-2016. Nutrients. 2019;11(10):2335.

40. Jeong YM, Dias C, Diekman C, Brochon H, Kim P, Kaur M, et al. Magnesium regulates the circadian oscillator in cyanobacteria. Journal of biological rhythms. 2019;34(4):380-90.

41. Abbasi B, Kimiagar M, Sadeghniiat K, Shirazi MM, Hedayati M, Rashidkhani B. The effect of magnesium supplementation on primary insomnia in elderly: A double-blind placebo-controlled clinical trial. Journal of research in medical sciences: the official journal of Isfahan University of Medical Sciences. 2012;17(12):1161. 
42. Sherman H, Gutman R, Chapnik N, Meylan J, Le Coutre J, Froy O. Caffeine alters circadian rhythms and expression of disease and metabolic markers. The international journal of biochemistry \& cell biology. 2011;43(5):829-38.

43. Grandner MA, Jackson N, Gerstner JR, Knutson KL. Sleep symptoms associated with intake of specific dietary nutrients. Journal of sleep research. 2014;23(1):22-34.

44. Liebman M, Chopin LF, Carter E, Clark AJ, Disney GW, Hegsted M, et al. Factors related to blood pressure in a biracial adolescent female population. Hypertension. 1986;8(10):843-50.

45. Torres-Farfan C, Serón-Ferré M, Dinet V, Korf HW. Immunocytochemical demonstration of day/night changes of clock gene protein levels in the murine adrenal gland: differences between melatoninproficient (C3H) and melatonin-deficient (C57BL) mice. Journal of pineal research. 2006;40(1):64-70.

46. St-Onge M-P, Mikic A, Pietrolungo CE. Effects of diet on sleep quality. Advances in Nutrition. 2016;7(5):938-49.

47. St-Onge M-P, Crawford A, Aggarwal B. Plant-based diets: reducing cardiovascular risk by improving sleep quality? Current sleep medicine reports. 2018;4(1):74-8.

48. Romain C, Alcaraz PE, Chung LH, Cases J. Regular consumption of HolisFiit, a polyphenol-rich extract-based food supplement, improves mind and body well-being of overweight and slightly obese volunteers: a randomized, double-blind, parallel trial. International journal of food sciences and nutrition. 2017;68(7):840-8.

49. Pengpid S, Peltzer K. Fruit and Vegetable Consumption is Protective from Short Sleep and Poor Sleep Quality Among University Students from 28 Countries. Nature and Science of Sleep. 2020;12:627.

50. Katagiri R, Asakura K, Kobayashi S, Suga H, Sasaki S. Low intake of vegetables, high intake of confectionary, and unhealthy eating habits are associated with poor sleep quality among middleaged female Japanese workers. Journal of occupational health. 2014:14-0051-0A.

51. Noorwali E, Hardie L, Cade J. Bridging the reciprocal gap between sleep and fruit and vegetable consumption: a review of the evidence, potential mechanisms, implications, and directions for future work. Nutrients. 2019;11(6):1382.

52. Hosseini A, Sobhanifar M-A, Forouzanfar F, Aghaee A, Rakhshandeh H. Hypnotic effect of red cabbage (Brassica oleracea) on pentobarbital-induced sleep in mice. Journal of pharmacy \& bioallied sciences. 2018;10(1):48.

53. Tanaka E, Yatsuya H, Uemura M, Murata C, Otsuka R, Toyoshima H, et al. Associations of protein, fat, and carbohydrate intakes with insomnia symptoms among middle-aged Japanese workers. Journal of epidemiology. 2013;23(2):132-8.

54. Del Brutto OH, Mera RM, Ha J-e, Gillman J, Zambrano M, Castillo PR. Dietary fish intake and sleep quality: a population-based study. Sleep medicine. 2016;17:126-8.

55. Dockray GJ. Cholecystokinin and gut-brain signalling. Regulatory peptides. 2009;155(1-3):6-10.

56. Wells AS, Read N, Uvnas-Moberg K, Alster P. Influences of fat and carbohydrate on postprandial sleepiness, mood, and hormones. Physiology \& behavior. 1997;61(5):679-86. 


\section{Tables}

Table 1, 2, 3 and 4 is not available with this version. 